\title{
High-order-harmonic generation in homonuclear and heteronuclear diatomic molecules: Exploration of multiple orbital contributions
}

\author{
John Heslar, ${ }^{1}$ Dmitry Telnov, ${ }^{2}$ and Shih-I Chu ${ }^{1,3}$ \\ ${ }^{1}$ Center for Quantum Science and Engineering, Department of Physics, National Taiwan University, Taipei 10617, Taiwan \\ ${ }^{2}$ Department of Physics, St. Petersburg State University, 198504 St. Petersburg, Russia \\ ${ }^{3}$ Department of Chemistry, University of Kansas, Lawrence, Kansas 66045, USA
}

(Received 18 January 2011; published 22 April 2011)

\begin{abstract}
We present a time-dependent density functional theory (TDDFT) approach with proper asymptotic long-range potential for nonperturbative treatment of high-order harmonic generation (HHG) of diatomic molecules with their molecular axis parallel to the laser field polarization. A time-dependent two-center generalized pseudospectral method in prolate spheroidal coordinate system is used for accurate and efficient treatment of the TDDFT equations in space and time. The theory is applied to a detailed all-electron nonperturbative investigation of HHG processes of homonuclear $\left(\mathrm{N}_{2}\right.$ and $\left.\mathrm{F}_{2}\right)$ and heteronuclear $(\mathrm{CO}, \mathrm{BF}$, and $\mathrm{HF})$ molecules in intense ultrashort laser pulses with the emphasis on the role of multiple molecular orbitals (MOs). The results reveal intriguing and substantially different nonlinear optical response behaviors for homonuclear and heteronuclear molecules. In particular, we found that the HHG spectrum for homonuclear molecules features a destructive interference of MO contributions while heteronuclear molecules show mostly constructive interference of orbital contributions.

DOI: 10.1103/PhysRevA.83.043414

PACS number(s): 33.80.Rv, 42.65.Ky, 31.15.E-, 31.15.A-
\end{abstract}

\section{INTRODUCTION}

The study of atomic and molecular processes in intense ultrashort laser fields is a subject of much current interest in science and technology [1]. In particular, high-order harmonic generation (HHG) is one of the hottest topics in strong-field atomic and molecular physics today, which is closely related to the recent advancements of attosecond science [1-3] as well as the frequency comb laser technology [4-7]. To describe such strong-field processes using fully $a b$ initio wave-function approach, it is necessary to solve the $(3 n+1)$-dimensional time-dependent Schrödinger equation (TDSE) in space and time, where $n$ is the number of electrons. This is well beyond the capability of current supercomputer technology when $n>2$. Even for the two-electron $(n=2)$ case, high-precision fully $a b$ initio $6 \mathrm{D}$ study of the HHG of the He atoms was achieved only recently by means of the time-dependent generalized pseudospectral approach in hyperspherical coordinates [8]. For many-electron molecular $(n \geqslant 2)$ systems, we have recently extended the time-dependent density functional theory (TDDFT) with proper asymptotic long-range potential [9-11] for the nonperturbative treatment of multiphoton ionization (MPI) and HHG processes of homonuclear and heteronuclear ( $\mathrm{CO}$ and $\mathrm{N}_{2}$ ) diatomic molecules in intense laser fields [12]. We have also applied our TDDFT approach to the study of MPI and HHG of diatomic molecules with arbitrary molecular axis orientation $[11,13,14]$, a subject of much current experimental interest. In our previous studies of MPI of homonuclear diatomic molecules $\mathrm{N}_{2}, \mathrm{O}_{2}$, and $\mathrm{F}_{2}$ and heteronuclear molecules $\mathrm{CO}$, we found that the highest occupied molecular orbital (HOMO) is not necessarily the dominant channel contributing to the strong-field molecular ionization $[9,12,13]$. For example, in the case of $F_{2}$ with the parallel orientation of the molecular axis with respect to the laser field polarization, the dominant ionization channel in strong fields is that associated with the $3 \sigma_{g}$ MO [9] while the HOMO is $1 \pi_{g}$. Furthermore, the ac Stark shift of individual MO and the detailed molecular electronic structure must be taken into account for a proper and quantitative treatment of the intense field atomic and molecular processes. This cannot be achieved by the models based on the strong field approximation (SFA) (see, e.g., [15-17] and references therein) for such models usually consider only the HOMO contribution and neglect the dynamic multielectron effects. On the other hand, TDDFT includes the multielectron effects through the exchange-correlation functional and all the spin orbitals, allowing the detailed study of the effect of multiple MOs on the MPI and HHG processes. With good quality exchange-correlation potentials (such as $\mathrm{LB} \alpha$ potential [18]), TDDFT has proved to provide quite accurate description of multielectron dynamics (for example, influence of the Cooper minimum on HHG in Ar atoms [11]).

In this paper, we extend the TDDFT with proper long-range potentials to the study of multi-electron heteronuclear and homonuclear diatomic molecules $\left(\mathrm{CO}, \mathrm{BF}, \mathrm{HF}, \mathrm{N}_{2}\right.$, and $\mathrm{F}_{2}$ ) with an aim to explore the nonlinear response of individual molecular orbitals (MO) to the laser field and their dynamic role in formation of the HHG spectra. We analyze the effect of asymmetry of the heteronuclear molecules on their HHG spectra, a subject of largely unexplored area of intense field molecular physics. In contrast with the homonuclear molecules which generate only odd harmonics of the laser frequency, aligned heteronuclear molecules can produce even harmonics as well [12]. In the high-energy part of the HHG spectra, the interference of contributions from different MO is mostly constructive for the heteronuclear molecules and destructive for the homonuclear molecules. We present an all-electron nonperturbative investigation of the HHG mechanisms taking into account the detailed electronic structure and the responses of individual electrons and using $\mathrm{N}_{2}, \mathrm{~F}_{2}, \mathrm{HF}, \mathrm{CO}$, and $\mathrm{BF}$ as examples $\left(\mathrm{N}_{2}, \mathrm{CO}\right.$, and $\mathrm{BF}$ are isoelectronic molecules with 14 electrons). The ground state electronic configurations of $\mathrm{N}_{2}$ and $\mathrm{F}_{2}$ (homonuclear molecules) are $1 \sigma_{g}^{2} 1 \sigma_{u}^{2} 2 \sigma_{g}^{2} 2 \sigma_{u}^{2} 1 \pi_{u}^{4} 3 \sigma_{g}^{2}$ and $1 \sigma_{g}^{2} 1 \sigma_{u}^{2} 2 \sigma_{g}^{2} 2 \sigma_{u}^{2} 3 \sigma_{g}^{2} 1 \pi_{u}^{4} 1 \pi_{g}^{4}$, respectively. The heteronuclear molecules $\mathrm{BF}$ and $\mathrm{CO}$ have the same ground state 
electronic configuration of $1 \sigma^{2} 2 \sigma^{2} 3 \sigma^{2} 4 \sigma^{2} 1 \pi^{4} 5 \sigma^{2}$, and for the HF molecule its ground state configuration appears as $1 \sigma^{2} 2 \sigma^{2} 3 \sigma^{2} 1 \pi^{4}$.

The organization of this paper is as follows. In Sec. II we briefly describe the TDDFT formalism for the general treatment of the multiphoton dynamics of heteronuclear and homonuclear diatomic molecular systems. In Sec. III we analyze the HHG spectra of the diatomic molecules and investigate the role of the contributions from different molecular orbitals. We identify the patterns of constructive and destructive interference of the orbital contributions to the total HHG spectrum. In the high-energy part of the spectrum, the interference appears destructive for the homonuclear molecules and constructive for the heteronuclear molecules. Section IV contains concluding remarks.

\section{TIME-DEPENDENT DFT FOR NONPERTURBATIVE TREATMENT OF DIATOMIC MOLECULES IN STRONG LASER FIELDS}

The basic equations of TDDFT are the time-dependent oneelectron Kohn-Sham equations for spin orbitals $\psi_{i \sigma}(\boldsymbol{r}, t)$ which involve an effective potential $v_{\text {eff, } \sigma}(\boldsymbol{r}, t)$ (in atomic units),

$$
\begin{aligned}
i \frac{\partial}{\partial t} \psi_{i \sigma}(\boldsymbol{r}, t) & =\left[-\frac{1}{2} \nabla^{2}+v_{\mathrm{eff}, \sigma}(\boldsymbol{r}, t)\right] \psi_{i \sigma}(\boldsymbol{r}, t), \\
i & =1,2, \ldots, N_{\sigma},
\end{aligned}
$$

where $N_{\sigma}\left(=N_{\uparrow}\right.$ or $\left.N_{\downarrow}\right)$ is the total number of electrons for a given spin $\sigma$, and the total number of electrons in the system is $N=\sum_{\sigma} N_{\sigma}$. The time-dependent effective potential $v_{\text {eff, } \sigma}(\boldsymbol{r}, t)$ is a functional of the electron spin-densities $\rho_{\sigma}(\boldsymbol{r}, t)$ which are related to the spin orbitals as follows:

$$
\rho_{\sigma}(\boldsymbol{r}, t)=\sum_{i=1}^{N_{\sigma}}\left|\psi_{i \sigma}(\boldsymbol{r}, t)\right|^{2}
$$

(the summation includes all spin orbitals with the same spin). The effective potential $v_{\text {eff, } \sigma}(\boldsymbol{r}, t)$ in Eq. (1) can be written in the following general form:

$$
v_{\mathrm{eff}, \sigma}(\boldsymbol{r}, t)=v_{\mathrm{H}}(\boldsymbol{r}, t)+v_{\mathrm{ext}}(\boldsymbol{r}, t)+v_{\mathrm{xc}, \sigma}(\boldsymbol{r}, t),
$$

where

$$
v_{\mathrm{H}}(\boldsymbol{r}, t)=\int \frac{\rho\left(\boldsymbol{r}^{\prime}, t\right)}{\left|\boldsymbol{r}-\boldsymbol{r}^{\prime}\right|} d \boldsymbol{r}^{\prime}
$$

is the Hartree potential due to electron-electron Coulomb interaction and $\rho(\boldsymbol{r}, t)$ is the total electron density

$$
\rho(\boldsymbol{r}, t)=\sum_{\sigma} \rho_{\sigma}(\boldsymbol{r}, t)
$$

$v_{\text {ext }}(\boldsymbol{r}, t)$ is the "external" potential due to the interaction of the electron with the external laser field and the nuclei. In the case of homonuclear or heteronuclear diatomic molecules in a linearly polarized external laser field, we have

$$
v_{\mathrm{ext}}(\boldsymbol{r}, t)=-\frac{Z_{1}}{\left|\boldsymbol{R}_{1}-\boldsymbol{r}\right|}-\frac{Z_{2}}{\left|\boldsymbol{R}_{2}-\boldsymbol{r}\right|}+[\boldsymbol{E}(t) \cdot \boldsymbol{r}] \sin \omega t,
$$

where $\boldsymbol{r}$ is the electronic coordinate, $E(t)$ is the electric field amplitude where the laser field is polarized along the molecular axis, $\boldsymbol{R}_{1}$ and $\boldsymbol{R}_{2}$ are the coordinates of the two nuclei at their fixed equilibrium positions, and $Z_{1}$ and $Z_{2}$ are the electric charges of the two nuclei, respectively. The internuclear separation $R$ is equal to $\left|\boldsymbol{R}_{2}-\boldsymbol{R}_{1}\right|$. Finally, $v_{\mathrm{xc}, \sigma}(\boldsymbol{r}, t)$ is the time-dependent exchange-correlation (xc) potential. Since the exact form of $v_{\mathrm{xc}, \sigma}(\boldsymbol{r}, t)$ is unknown, the adiabatic approximation is often used $[19,20]$

$$
v_{\mathrm{xc}, \sigma}(\boldsymbol{r}, t)=\left.v_{\mathrm{xc}, \sigma}\left[\rho_{\sigma}\right]\right|_{\rho_{\sigma}=\rho_{\sigma}(\boldsymbol{r}, t)} .
$$

When these potentials, determined by the time-independent ground-state density functional theory (DFT) are used along with TDDFT in the electronic structure calculations, both inner shell and excited states can be calculated rather accurately [21]. In this work, we utilize the improved van Leeuwen-Baerends $\mathrm{LB} \alpha$ xc potential [18]. The $\mathrm{LB} \alpha$ contains two empirical parameters $\alpha$ and $\beta$ and has the following explicit form in the adiabatic approximation:

$$
\begin{aligned}
v_{\mathrm{xc}, \sigma}^{\mathrm{LB} \alpha}(\boldsymbol{r}, t) & \alpha v_{\mathrm{x}, \sigma}^{\mathrm{LSDA}}(\boldsymbol{r}, t)+v_{\mathrm{c}, \sigma}^{\mathrm{LSDA}}(\boldsymbol{r}, t) \\
& -\frac{\beta x_{\sigma}^{2}(\boldsymbol{r}, t) \rho_{\sigma}^{1 / 3}(\boldsymbol{r}, t)}{1+3 \beta x_{\sigma}(\boldsymbol{r}, t) \ln \left\{x_{\sigma}(\boldsymbol{r}, t)+\left[x_{\sigma}^{2}(\boldsymbol{r}, t)+1\right]^{1 / 2}\right\}} .
\end{aligned}
$$

Here $\rho_{\sigma}$ is the electron density with spin $\sigma$, and we use $\alpha=1.19$ and $\beta=0.01$ [22]. The first two terms in Eq. (8) $\left(v_{\mathrm{x}, \sigma}^{\mathrm{LSDA}}\right.$ and $\left.v_{\mathrm{c}, \sigma}^{\mathrm{LSDA}}\right)$ are the LSDA exchange and correlation potentials that do not have the correct Coulombic asymptotic behavior. The last term in Eq. (8) is the nonlocal gradient correction with $x_{\sigma}(\boldsymbol{r})=\left|\nabla \rho_{\sigma}(\boldsymbol{r})\right| / \rho_{\sigma}^{4 / 3}(\boldsymbol{r})$, which ensures the proper long-range Coulombic asymptotic potential $v_{\mathrm{xc}, \sigma}^{\mathrm{LB} \alpha} \rightarrow$ $-1 / r$ as $r \rightarrow \infty$. Note that if the conventional xc energy functional forms taken from local spin density approximation (LSDA) or generalized gradient approximation (GGA) $[23,24]$ are used, the corresponding xc potential $v_{\mathrm{xc}, \sigma}(\boldsymbol{r}, t)$ will not possess the correct long-range asymptotic $(-1 / r)$ behavior [25]. For the time-independent case, this exchange-correlation $\mathrm{LB} \alpha$ potential has been found to be reliable for atomic and molecular DFT calculations $[9,10,18,26]$.

For the numerical solution of the TDDFT equations for diatomic molecules with proper long-range potential, we have recently developed a time-dependent generalized pseudospectral (TDGPS) method in prolate spheroidal coordinate system $[9,12,27]$. The advantage of this method is that it allows nonuniform and optimal spatial grid discretization (denser mesh near each nucleus and sparser mesh at larger electron-nucleus separations). This improves greatly both the accuracy and the efficiency of the electronic structure and time-dependent calculations with the use of only a modest number of grid points. In this work we extend this procedure to the numerical solution of the TDDFT calculations for the twocenter homonuclear and heteronuclear diatomic molecular systems in the presence of intense laser fields.

Table I lists the MO energies calculated with the $\mathrm{LB} \alpha$ potential using 50 grid points in $\xi$ and 30 grid points in $\eta$. The agreement of the calculated valence MO energies with the experimental data is well within 0.01 a.u. 
TABLE I. Comparison of the field-free molecular orbital energy levels of $\mathrm{F}_{2}, \mathrm{BF}, \mathrm{CO}, \mathrm{N}_{2}$, and $\mathrm{HF}$ calculated with the $\mathrm{LB} \alpha$ potential and the experimental ionization potentials (in a.u.).

\begin{tabular}{|c|c|c|c|c|c|c|c|}
\hline \multicolumn{8}{|c|}{$\mathrm{F}_{2}$} \\
\hline Orbital & $1 \sigma_{g}$ & $1 \sigma_{u}$ & $2 \sigma_{g}$ & $2 \sigma_{u}$ & $3 \sigma_{g}$ & $1 \pi_{u}$ & $1 \pi_{g}$ \\
\hline Expt. [28] & 25.601 & 25.609 & 1.5342 & 1.377 & 0.775 & 0.691 & 0.5832 \\
\hline $\mathrm{LB} \alpha$ & 25.1531 & 25.1531 & 1.4508 & 1.2252 & 0.7907 & 0.6939 & 0.5739 \\
\hline \multicolumn{8}{|c|}{$\mathrm{BF}$} \\
\hline Orbital & $1 \sigma$ & $2 \sigma$ & $3 \sigma$ & $4 \sigma$ & $1 \pi$ & $5 \sigma$ & \\
\hline Expt. $[29,30]$ & & & & & & 0.4085 & \\
\hline $\mathrm{LB} \alpha$ & 25.1669 & 7.1745 & 1.4140 & 0.7763 & 0.6970 & 0.3895 & \\
\hline \multicolumn{8}{|c|}{$\mathrm{CO}$} \\
\hline Orbital & $1 \sigma$ & $2 \sigma$ & $3 \sigma$ & $4 \sigma$ & $1 \pi$ & $5 \sigma$ & \\
\hline Expt. [31] & 19.9367 & 10.8742 & 1.3964 & 0.7239 & 0.6247 & 0.5144 & \\
\hline $\mathrm{LB} \alpha$ & 19.6142 & 10.6556 & 1.2549 & 0.7071 & 0.6276 & 0.5086 & \\
\hline \multicolumn{8}{|c|}{$\mathrm{N}_{2}$} \\
\hline Orbital & $1 \sigma_{g}$ & $1 \sigma_{u}$ & $2 \sigma_{g}$ & $2 \sigma_{u}$ & $1 \pi_{u}$ & $3 \sigma_{g}$ & \\
\hline Expt. [32-34] & 15.0492 & 15.0492 & 1.3708 & 0.6883 & 0.6233 & 0.5726 & \\
\hline $\mathrm{LB} \alpha$ & 14.7962 & 14.7950 & 1.2162 & 0.6786 & 0.6199 & 0.5682 & \\
\hline \multicolumn{8}{|c|}{$\mathrm{HF}$} \\
\hline Orbital & $1 \sigma$ & $2 \sigma$ & $3 \sigma$ & $1 \pi$ & & & \\
\hline Expt. [35] & 25.5132 & 1.4545 & 0.7284 & 0.5898 & & & \\
\hline $\mathrm{LB} \alpha$ & 25.0786 & 1.3074 & 0.7070 & 0.5741 & & & \\
\hline
\end{tabular}

\section{HIGH-ORDER HARMONIC GENERATION OF HETERONUCLEAR AND HOMONUCLEAR DIATOMIC MOLECULES IN INTENSE LASER FIELDS}

The time-dependent induced dipole moment is calculated by the total electron density $\rho(\boldsymbol{r}, t)$,

$$
d(t)=\int z \rho(\boldsymbol{r}, t) d \boldsymbol{r}=\sum_{i \sigma} d_{i \sigma}(t),
$$

where

$$
d_{i \sigma}(t)=n_{i \sigma}\left\langle\psi_{i \sigma}(\boldsymbol{r}, t)|z| \psi_{i \sigma}(\boldsymbol{r}, t)\right\rangle
$$

is the induced dipole moment of the $i \sigma$ th spin orbital and $n_{i \sigma}$ is its electron occupation number. The power spectrum of the HHG is then acquired by taking the Fourier transform of the total time-dependent induced dipole moment $d(t)$ :

$$
P(\omega)=\left|\frac{1}{t_{f}-t_{i}} \int_{t_{i}}^{t_{f}} d(t) e^{-i \omega t} d t\right|^{2} .
$$

For the CO molecule the HOMO $(5 \sigma)$ is dominant for the whole HHG spectrum (Fig. 1); other orbitals contribute much less. The distinct harmonic peaks are seen up to the order 30. In $\mathrm{CO}$ the $5 \sigma$ (HOMO) permanent dipole is 1.57 a.u. which is much larger than that of the other orbital. The density for the $5 \sigma$ (and $2 \sigma$ ) is mostly localized on the carbon atom. For all other orbitals the density is localized on the oxygen atom. Looking at the time-dependent orbital dipoles for the CO molecule (Fig. 2), we can see that all other orbitals that are localized on the oxygen atom are in phase and $5 \sigma$ (carbon) orbital is out of phase in time.
The $\mathrm{N}_{2}$ molecule has dipole amplitudes that follow the trend

$$
d_{2 \sigma_{g}}<d_{1 \pi_{u}}<d_{\text {total }}<d_{3 \sigma_{g}}<d_{2 \sigma_{u}} .
$$

The orbital dipole moments $2 \sigma_{g}, 1 \pi_{u}$, and $2 \sigma_{u}$ are oscillating with the same sign (in phase) of the total dipole, the $3 \sigma_{g}$ has opposite sign (out of phase). So when we look at the two orbital dipole amplitudes $2 \sigma_{u}$ and $3 \sigma_{g}$ with similar intensities, they
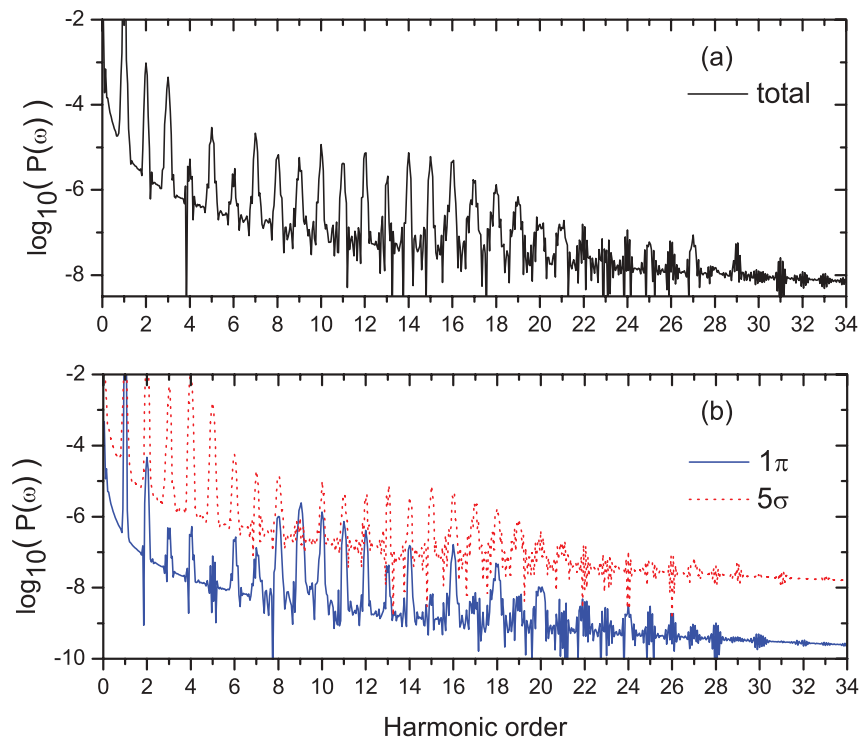

FIG. 1. (Color online) Total (a) and orbital (b) harmonic power spectra of the $\mathrm{CO}$ molecule in the $\sin ^{2}$ laser pulse with a peak intensity of $I_{0}=1 \times 10^{14} \mathrm{~W} / \mathrm{cm}^{2}$. The laser pulse has a wavelength of $800 \mathrm{~nm}$ and a time duration of 20 optical cycles. 


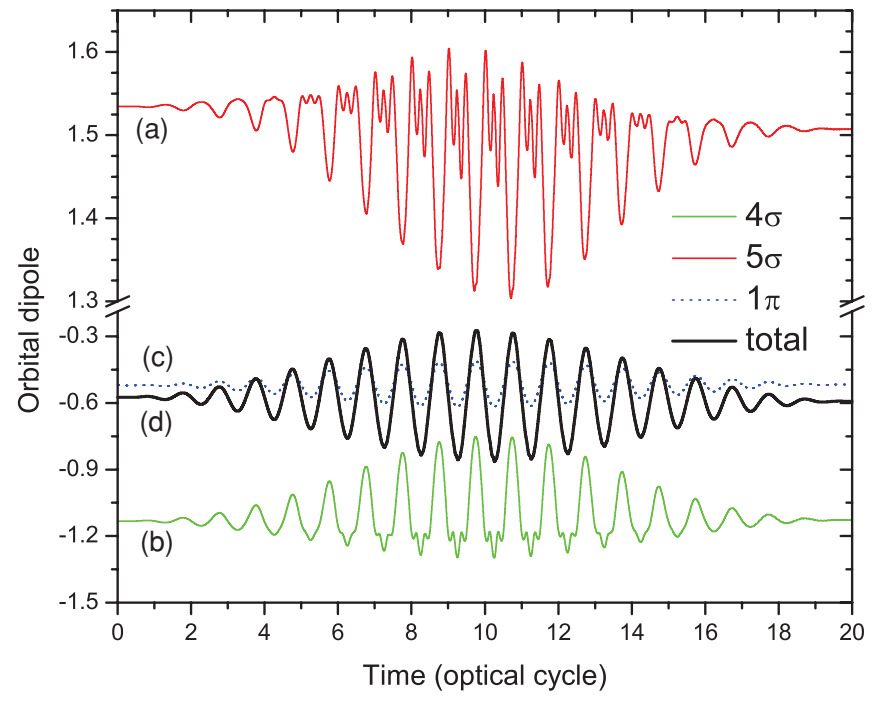

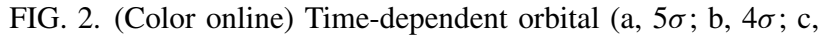
$1 \pi)$ and total $(\mathrm{d})$ dipole moments of the CO molecule in the $\sin ^{2}$ laser pulse with a peak intensity of $I_{0}=1 \times 10^{14} \mathrm{~W} / \mathrm{cm}^{2}$. The laser pulse has a wavelength of $800 \mathrm{~nm}$ and a time duration of 20 optical cycles. All dipoles are in a.u.

oscillate in time with different sign, canceling each other out (Fig. 3), leading to a smaller total dipole.

The behavior of the HHG spectrum (Fig. 4) for $\mathrm{N}_{2}$ is quite different than that of a heteronuclear diatomic molecule. The HOMO $\left(3 \sigma_{g}\right)$ is dominant in the middle part of the spectrum (again up to the order 31). However, starting the 33th harmonic, the $2 \sigma_{u}$ orbital has a comparable contribution which interferes destructively with that of $3 \sigma_{g}$. Thus the result of interference is much lower than both of the single orbital spectra. Then this two-orbital spectrum becomes comparable in magnitude with the $2 \sigma_{g}$-only HHG. Again the destructive contributions takes place and the result is much lower than any of the single orbital

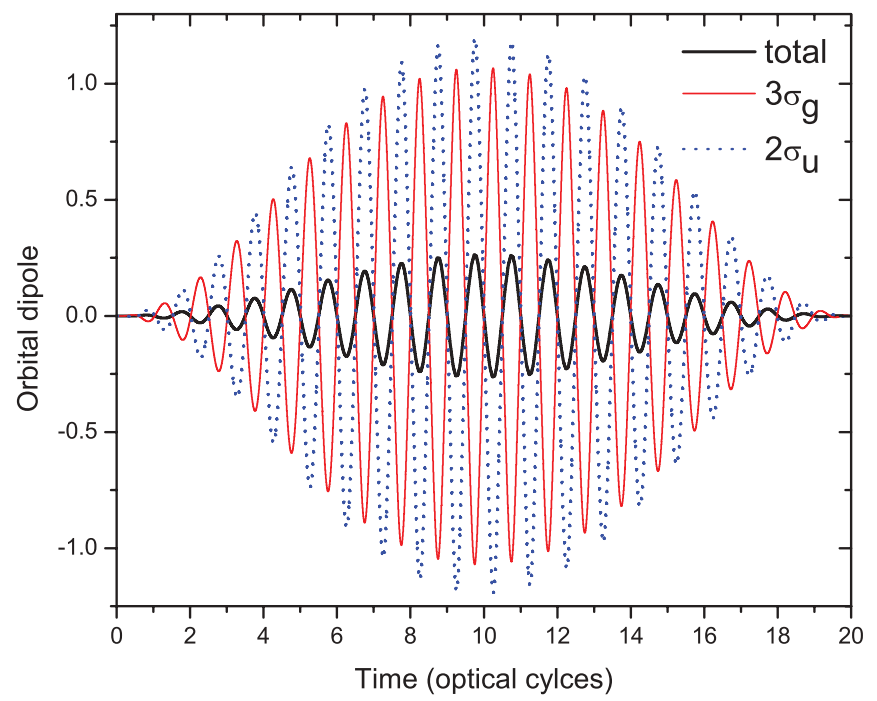

FIG. 3. (Color online) Time-dependent orbital and total dipole moments of the $\mathrm{N}_{2}$ molecule in the $\sin ^{2}$ laser pulse with a peak intensity of $I_{0}=1 \times 10^{14} \mathrm{~W} / \mathrm{cm}^{2}$. The laser pulse has a wavelength of $800 \mathrm{~nm}$ and a time duration of 20 optical cycles. All dipoles are in a.u.
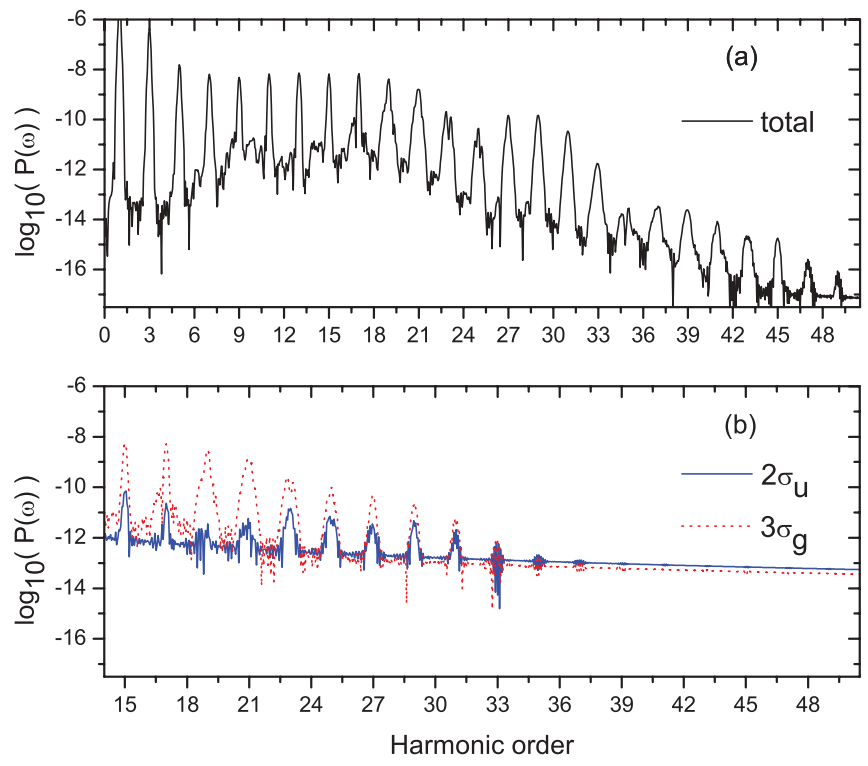

FIG. 4. (Color online) Total (a) and orbital (b) harmonic power spectra of the $\mathrm{N}_{2}$ molecule in the $\sin ^{2}$ laser pulse with a peak intensity of $I_{0}=1 \times 10^{14} \mathrm{~W} / \mathrm{cm}^{2}$. The laser pulse has a wavelength of $800 \mathrm{~nm}$ and a time duration of 20 optical cycles.

contributions. In summary, we can say that the long HHG spectrum of $\mathrm{N}_{2}$ is a collective multielectron effect. Destructive interference between $2 \sigma_{u}$ and $3 \sigma_{g}$ orbital contributions makes the resulting total HHG spectrum lower in amplitude with distinct harmonic peaks up to the order 49.

In contrast to the $\mathrm{N}_{2}$ molecule, the full destructive interference is not possible in $\mathrm{CO}$ because of the broken $g-u$ symmetry. The induced dipole moment of the HOMO $5 \sigma$ orbital is so large that no other orbital can make a comparable contribution. To prove this point about heteronuclear and homonuclear diatomic molecules we look at $\mathrm{F}_{2}, \mathrm{BF}$, and the

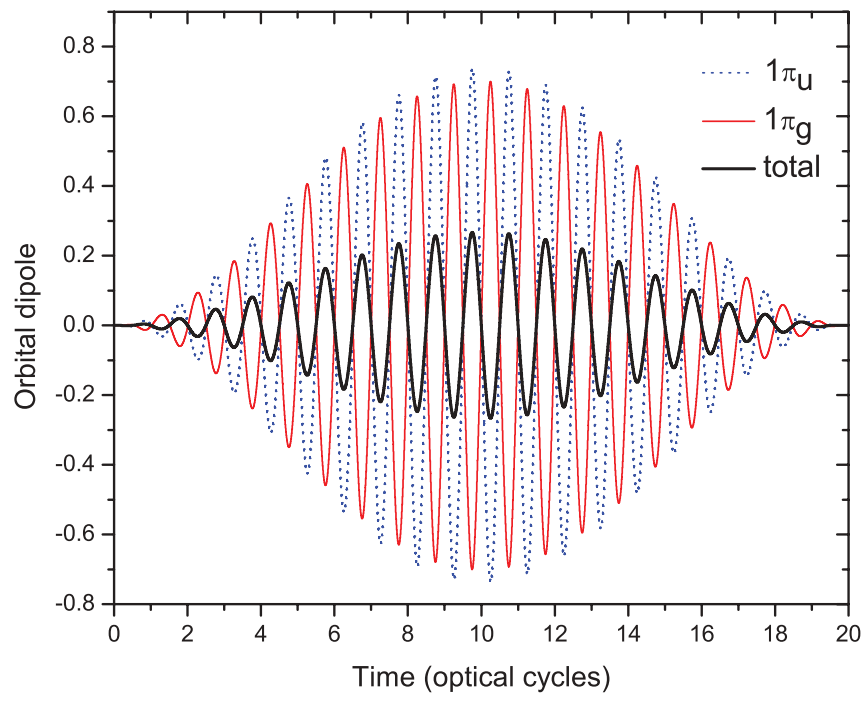

FIG. 5. (Color online) Time-dependent orbital and total dipole moments of the $F_{2}$ molecule in the $\sin ^{2}$ laser pulse with a peak intensity of $I_{0}=1 \times 10^{14} \mathrm{~W} / \mathrm{cm}^{2}$. The laser pulse has a wavelength of $800 \mathrm{~nm}$ and a time duration of 20 optical cycles. All dipoles are in a.u. 

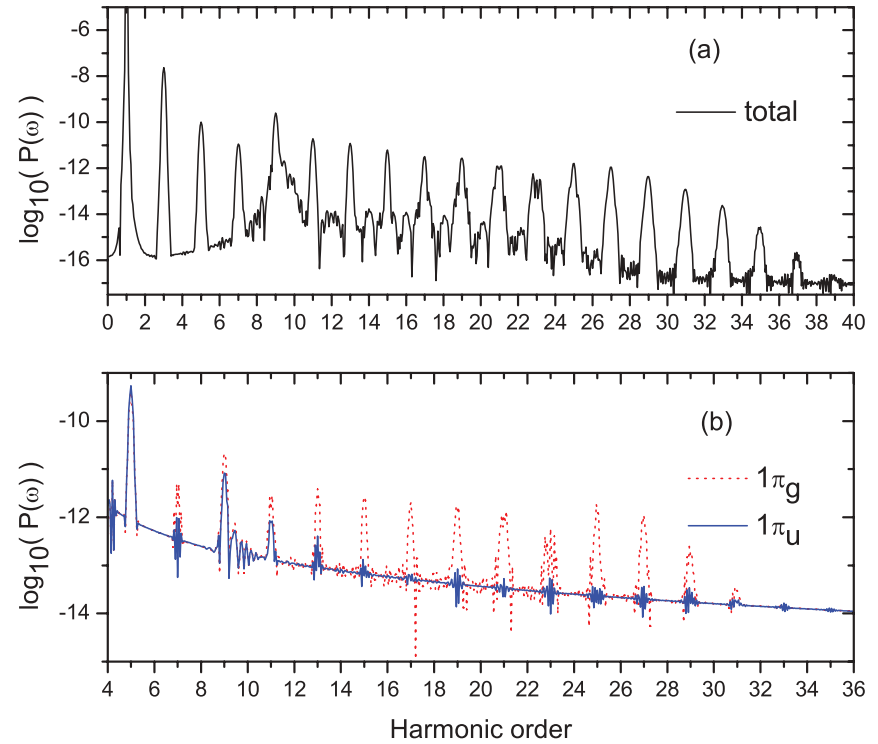

FIG. 6. (Color online) Total (a) and orbital (b) harmonic power spectra of the $\mathrm{F}_{2}$ molecule in the $\sin ^{2}$ laser pulse with a peak intensity of $I_{0}=1 \times 10^{14} \mathrm{~W} / \mathrm{cm}^{2}$. The laser pulse has a wavelength of $800 \mathrm{~nm}$ and a time duration of 20 optical cycles.

heteronuclear molecule that has the greatest dipole moment, HF. Here we can see if these molecules exhibit similar properties of $\mathrm{CO}$ and $\mathrm{N}_{2}$. We see the same characteristics in $\mathrm{F}_{2}$ as we can see in $\mathrm{N}_{2}$. The orbital dipole moments of $2 \sigma_{u}$ and $1 \pi_{g}$ are oscillating out of phase, from other orbitals, including the total. So when we look at the two orbital dipole amplitudes $1 \pi_{u}$ and $1 \pi_{g}$, they oscillate in time with different sign, canceling each other out (Fig. 5), leading to a smaller total dipole. The behavior of the HHG spectrum of $\mathrm{F}_{2}$ is similar to that of $\mathrm{N}_{2}$. If we look at Fig. 6, the $1 \pi_{u}$ and $1 \pi_{g}$ orbitals dominate the HHG spectrum, and it has much greater intensity than that of the total HHG. In Fig. 6 we see there is destructive interference

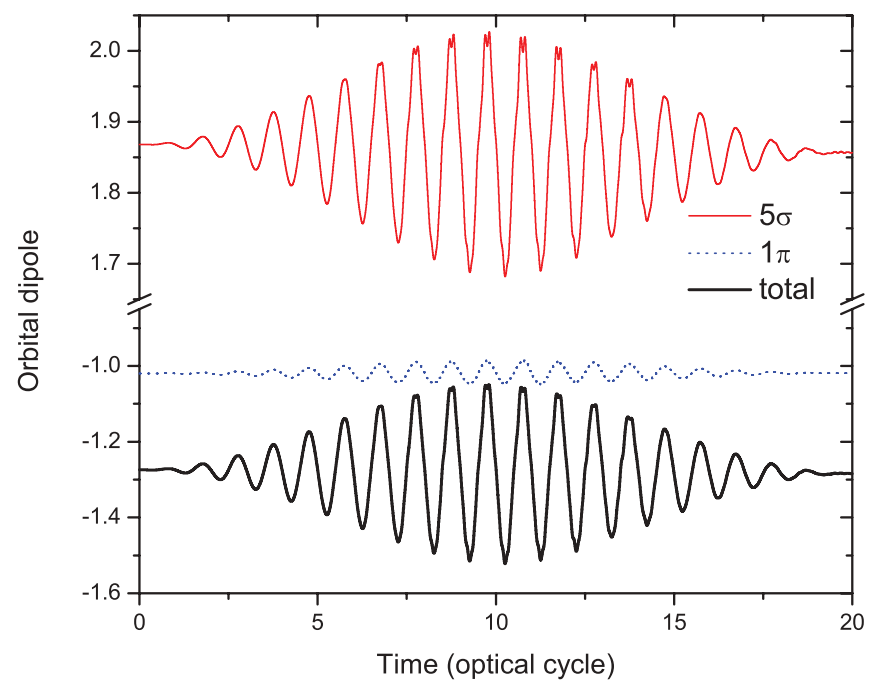

FIG. 7. (Color online) Time-dependent orbital and total dipole moments of the $\mathrm{BF}$ molecule in the $\sin ^{2}$ laser pulse with a peak intensity of $I_{0}=1 \times 10^{14} \mathrm{~W} / \mathrm{cm}^{2}$. The laser pulse has a wavelength of $800 \mathrm{~nm}$ and a time duration of 20 optical cycles. All dipoles are in a.u.
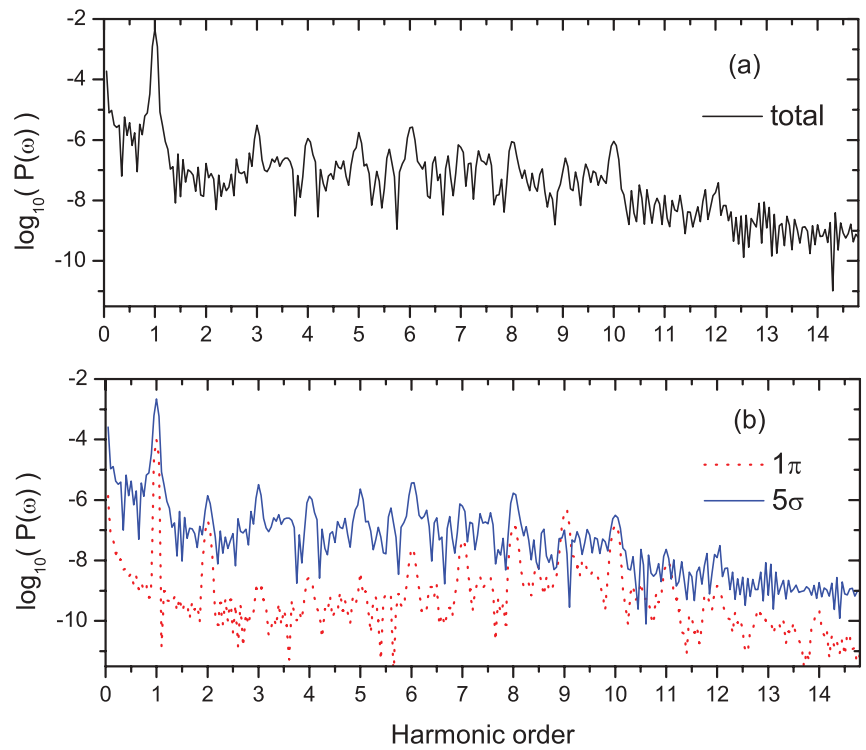

FIG. 8. (Color online) Total (a) and orbital (b) harmonic power spectra of the BF molecule in the $\sin ^{2}$ laser pulse with a peak intensity of $I_{0}=1 \times 10^{14} \mathrm{~W} / \mathrm{cm}^{2}$. The laser pulse has a wavelength of $800 \mathrm{~nm}$ and a time duration of 20 optical cycles.

between the contributions from the $1 \pi_{u}$ and $1 \pi_{g}$ orbitals, we explained this previously by saying their dipole moments are out of phase with one another. Since these two orbitals $\left(1 \pi_{u}\right.$ and $\left.1 \pi_{g}\right)$ have the greatest amplitude of the induced dipole, they will have the greatest contributions to the power spectrum.

Previously we only looked at the CO molecule for the heteronuclear diatomic system, and saw where the HOMO dominates the HHG spectrum and dipole moment. Now we examine two other heteronuclear molecules (BF and $\mathrm{HF}$ ) and see if their characteristics resemble that of the $\mathrm{CO}$ molecule.

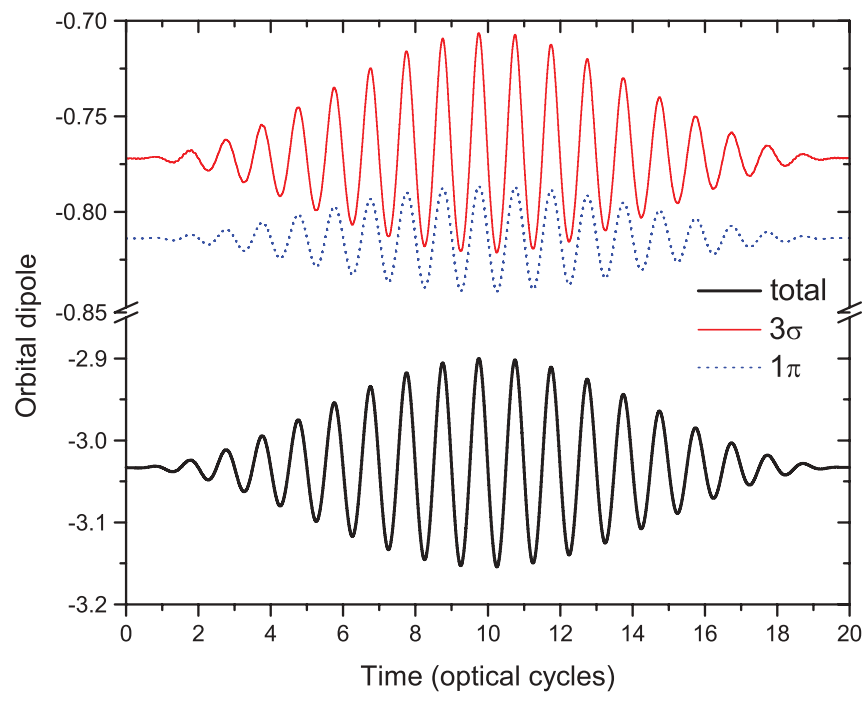

FIG. 9. (Color online) Time-dependent orbital and total dipole moments of the HF molecule in the $\sin ^{2}$ laser pulse with a peak intensity of $I_{0}=1 \times 10^{14} \mathrm{~W} / \mathrm{cm}^{2}$. The laser pulse has a wavelength of $800 \mathrm{~nm}$ and a time duration of 20 optical cycles. All dipoles are in a.u. 

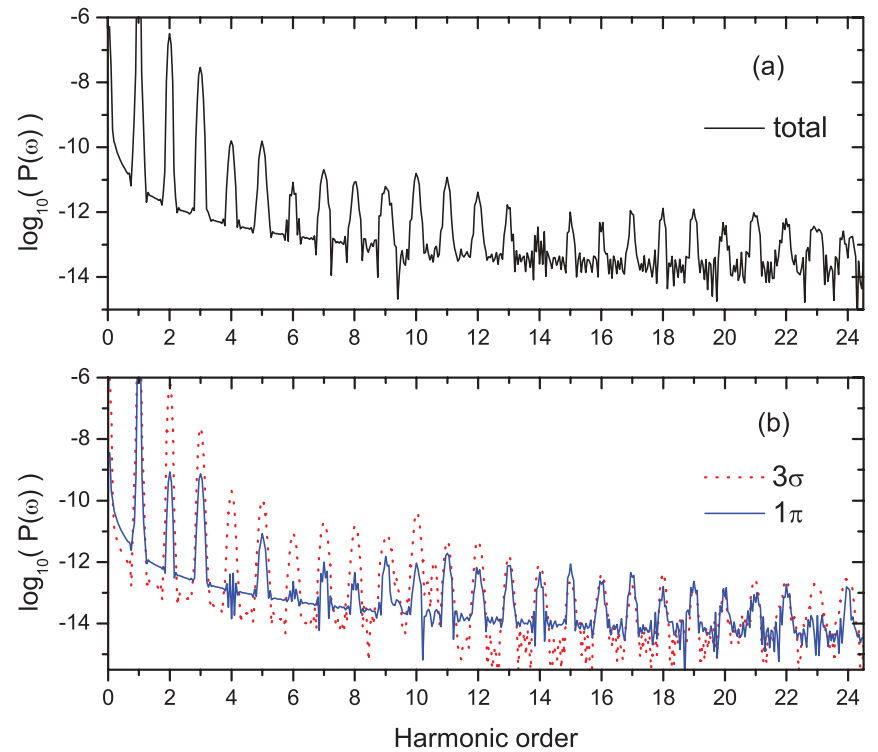

FIG. 10. (Color online) Total (a) and orbital (b) harmonic power spectra of the HF molecule in the $\sin ^{2}$ laser pulse with a peak intensity of $I_{0}=1 \times 10^{14} \mathrm{~W} / \mathrm{cm}^{2}$. The laser pulse has a wavelength of $800 \mathrm{~nm}$ and a time duration of 20 optical cycles.

To understand the HHG spectrum, again we look at the orbital dipole moment of BF. Figure 7 shows that the $5 \sigma$ orbital (like that of $\mathrm{CO}$, since they are isoelectronic) has the greatest dipole moment. This is understandable since most of the electronic density resides on the fluorine atom. The fluorine atom is more electronegative than that of the boron atom, so it will have the greater electron density. As previous seen in the $\mathrm{CO}$ molecule, $\mathrm{BF}$ has the same physical characteristics of its orbital HHG spectrum. Figure 8 shows that HOMO $(5 \sigma)$ has the greatest contribution to the total HHG.

Now we will study the heteronuclear molecule that has the greatest permanent dipole moment, and look at the physical characteristics of hydrogen fluoride. HF has a permanent dipole moment of $1.820 \mathrm{D}$, where most of the electron density resides on the fluorine atom. For HF molecule the HOMO is the $1 \pi$ orbital, and it has the greatest negative value of the orbital dipole moment (see Fig. 9). However, when we look at the orbital HHG spectrum (Fig. 10), we see the greatest orbital contribution to the HHG spectrum was from $3 \sigma$, not the HOMO orbital $(1 \pi)$. Since the field is linearly polarized to the $z$ axis and the $1 \pi$ orbital is perpendicular to the $z$ axis, the induced dipole moment is aligned not with the $1 \pi$ orbital but with the $3 \sigma$ orbital. We see between harmonic order peaks (even and odd), the $1 \pi$ orbital has greater intensity than that of the $3 \sigma$ orbital. At peak values (even and odd harmonic orders) the $3 \sigma$ orbital has almost the same intensity as that of the total HHG spectrum. We conclude that HF follows the same orbital characteristics of other heteronuclear molecules studied (CO and $\mathrm{BF}$ ).

\section{CONCLUSION}

In this article we have presented a detailed investigation and comparison of the very high-order nonlinear optical response of the homonuclear $\left(\mathrm{N}_{2}\right.$ and $\left.\mathrm{F}_{2}\right)$ and heteronuclear $(\mathrm{CO}, \mathrm{BF}$, and HF) diatomic molecules in intense ultrashort laser fields, with parallel orientation of the molecular axis with respect to the polarization of the laser field. We make use of TDDFT with a correct asymptotic long-range $(-1 / r)$ potential to ensure that the individual spin orbitals have the proper ionization potentials. Our analysis of the HHG spectra reveals that homonuclear molecules have destructive interference between the orbital contributions to the total harmonic signal. This happens because the induced dipole moments of different orbitals oscillate in time with opposite phases, so their contributions are canceled out in the total dipole moment. Therefore a close look at the induced dipole moment of the homonuclear diatomic molecules can give predictions of what orbital contributions will interfere destructively in the total HHG spectrum. The destructive interference in the HHG spectrum accounts for the unexpected observation that some of the individual orbitals harmonic power spectra have greater intensity than that of the total HHG. The HHG process by the heteronuclear diatomic molecules has a quite different characteristic. First, heteronuclear molecules can generate even and odd harmonics since they lack the inversion symmetry. Second, for all the studied heteronuclear molecules the HOMO contribution to the total HHG spectrum is by far dominant, and the total harmonic signal has almost the same intensity as that produced by HOMO only. The interference between the different orbitals in the total HHG spectrum is mostly constructive. The same features are observed in the time-dependent induced dipole moments, with the HOMO dipole moment having the largest oscillation magnitude.

\section{ACKNOWLEDGMENTS}

This work was partially supported by the Chemical Sciences, Geosciences and Biosciences Division of the Office of Basic Energy Sciences, Office of Sciences, US Department of Energy, and by the US National Science Foundation. We also acknowledge the partial support of National Science Council of Taiwan (Grant No. 97-2112-M-002-003-MY3) and National Taiwan University (Grants No. 98R0045 and No. 99R80870).
[1] F. Krausz and M. Ivanov, Rev. Mod. Phys. 81, 163 (2009).

[2] P. B. Corkum and F. Krausz, Nat. Phys. 3, 381 (2007).

[3] J. J. Carrera, X. M. Tong, and S. I. Chu, Phys. Rev. A 74, 023404 (2006).
[4] C. Gohle, T. Udem, M. Herrmann, J. Rauschenberger, R. Holzwarth, H. A. Schuessler, F. Krausz, and T. W. Hänsch, Nature (London) 436, 234 (2005).

[5] R. J. Jones, K. D. Moll, M. J. Thorpe, and J. Ye, Phys. Rev. Lett. 94, 193201 (2005). 
[6] J. J. Carrera, S.-K. Son, and S. I. Chu, Phys. Rev. A 77, 031401(R) (2008).

[7] J. J. Carrera and S. I. Chu, Phys. Rev. A 79, 063410 (2009).

[8] X. X. Guan, X. M. Tong, and S. I. Chu, Phys. Rev. A 73, 023403 (2006).

[9] X. Chu and S. I. Chu, Phys. Rev. A 70, 061402(R) (2004).

[10] S. I. Chu, J. Chem. Phys. 123, 062207 (2005).

[11] D. A. Telnov and S. I. Chu, Phys. Rev. A 80, 043412 (2009).

[12] J. Heslar, J. J. Carrera, D. A. Telnov, and S. I. Chu, Int. J. Quantum Chem. 107, 3159 (2007).

[13] D. A. Telnov and S. I. Chu, Phys. Rev. A 79, 041401(R) (2009).

[14] S. K. Son and S. I. Chu, Phys. Rev. A 80, 011403(R) (2009).

[15] Z. Chen, T. Morishita, A. T. Le, and C. D. Lin, Phys. Rev. A 76, 043402 (2007).

[16] S. Odžak and D. B. Milošević, Phys. Rev. A 79, 023414 (2009).

[17] V. I. Usachenko, P. E. Pyak, and V. V. Kim, Phys. Rev. A 79, 023415 (2009).

[18] P. R. T. Schipper, O. V. Gritsenko, S. J. A. van Gisbergen, and E. J. Baerends, J. Chem. Phys. 112, 1344 (2000).

[19] X. Chu and S. I. Chu, Phys. Rev. A 63, 023411 (2001).

[20] C. A. Ullrich, U. J. Gossmann, and E. K. U. Gross, Phys. Rev. Lett. 74, 872 (1995).

[21] M. A. L. Marques, C. A. Ullrich, F. Nogueira, A. Rubio, K. Burke, and E. K. U. Gross, Time-Dependent Density Functional Theory (Springer, Berlin, 2006).

[22] X. Chu and S. I. Chu, Phys. Rev. A 64, 063404 (2001).
[23] R. G. Parr and W. T. Yang, Density-Functional Theory of Atoms and Molecules (Oxford University Press, New York, 1989).

[24] R. M. Dreizler and E. K. U. Gross, Density Functional Theory, An Approach to Quantum Many-Body Probelm (Springer, Berlin, 1990).

[25] X. M. Tong and S. I. Chu, Phys. Rev. A 55, 3406 (1997).

[26] R. van Leeuwen and E. J. Baerends, Phys. Rev. A 49, 2421 (1994).

[27] D. A. Telnov and S. I. Chu, Phys. Rev. A 71, 013408 (2005).

[28] A. Lofthus and P. H. Krupenie, J. Phys. Chem. Ref. Data 6, 113 (1977).

[29] R. B. Caton and A. E. Douglas, Can. J. Phys. 48, 432 (1970).

[30] D. L. Hildenbrand, Int. J. Mass Spectrom. Ion Phys. 7, 255 (1971).

[31] K. Siegbahn, J. Electron Spectrosc. Relat. Phenom. 5, 3 (1974).

[32] G. Johansson, J. Hedman, A. Berndtsson, M. Klasson, and R. Nilsson, J. Electron Spectrosc. Relat. Phenom. 2, 295 (1973).

[33] H. Hamnett, W. Stoll, and C. E. Brion, J. Electron Spectrosc. Relat. Phenom. 8, 367 (1976).

[34] D. W. Turner, C. Baker, A. D. Baker, and C. R. Brundle, Molecular Photoelectron Spectroscopy (Wiley Interscience, London, 1970).

[35] R. W. Shaw Jr. and T. D. Thomas, Phys. Rev. A 11, 1491 (1975). 